CORRECTION

https://doi.org/10.1038/s41586-019-1084-8

\section{Author Correction: CasX enzymes comprise a distinct family of RNA-guided genome editors}

Jun-Jie Liu, Natalia Orlova, Benjamin L. Oakes, Enbo Ma, Hannah B. Spinner, Katherine L. M. Baney, Jonathan Chuck, Dan Tan, Gavin J. Knott, Lucas B. Harrington, Basem Al-Shayeb, Alexander Wagner, Julian Brötzmann, Brett T. Staahl, Kian L. Taylor, John Desmarais, Eva Nogales \& Jennifer A. Doudna

Correction to: Nature https://doi.org/10.1038/s41586-019-0908-x, published online 04 February 2019.

In this Article, there are issues concerning the tracing of the first 30 nucleotides within the single-guide RNA (sgRNA), which after careful inspection we conclude run in the opposite direction. We have fixed this error and used the opportunity to improve small segments of the CasX protein modelled in poorly defined peripheral regions. After revising the Protein Data Bank (PDB) coordinates, some minor changes were made to Figs. 3, 4 and Extended Data Fig. 6. The original and corrected Figs. 3, 4 and Extended Data Fig. 6 are shown as Figs. 1, 2 and 3 of this Amendment, for comparison and transparency. The corrected models (for the different states) have new PDB accession codes that should supersede the previous ones. As a result, the 'Data availability' statement and Supplementary Table 1 of the original Article, which contain the PDB accession information, have also been updated. In the 'Data availability' section, PDB codes 6E5O, 6E7A and 6E79 have been corrected to $6 \mathrm{NY} 1,6 \mathrm{NY} 2$ and $6 \mathrm{NY} 3$. In addition, the sgRNA cartoons have been updated in Supplementary Video 1 (the original video is shown as Supplementary Information to this Amendment, for transparency). None of these changes affects the conclusions of the Article, and the original Article has been corrected online.

Supplementary Information is available in the online version of this Amendment.
Original Fig. 3b

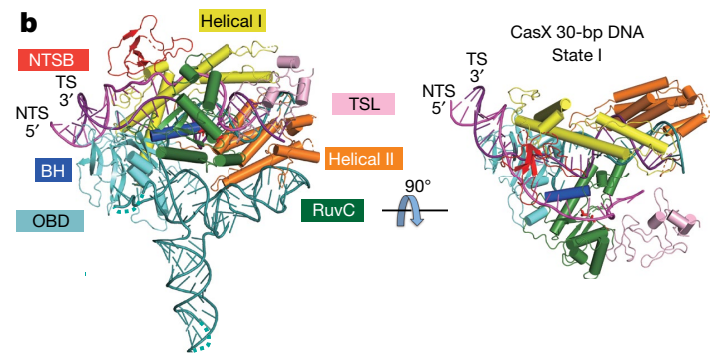

Corrected Fig. 3b

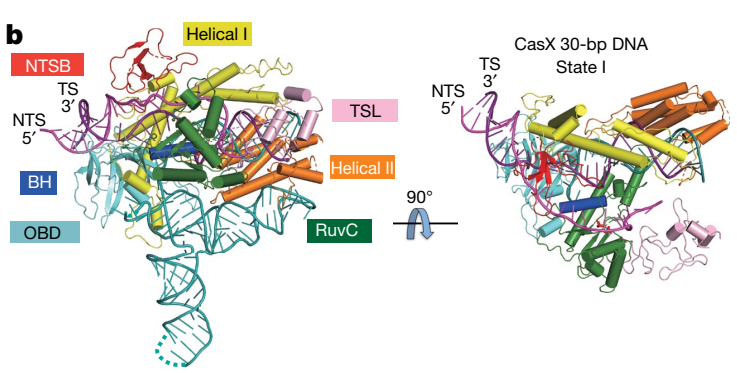

Original Fig. $3 f$

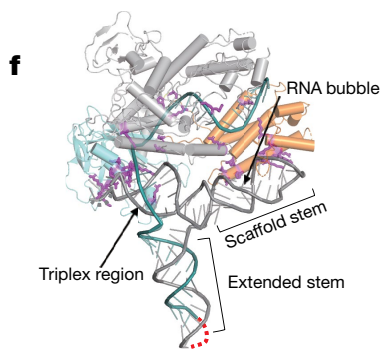

Corrected Fig. 3f

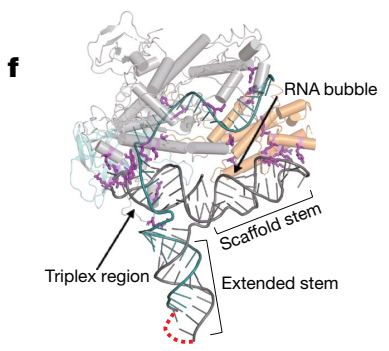

Fig. 1 This is the corrected Fig. $\mathbf{3 b}$, $\mathbf{f}$ and the incorrect Fig. $\mathbf{3 b}, \mathbf{f}$ published in the original Article. The CasX sgRNA presentations have been updated. 


\section{Original Fig. 4}

a

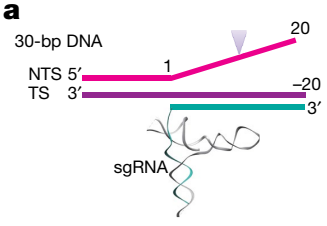

b

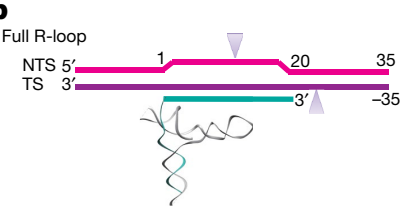

C

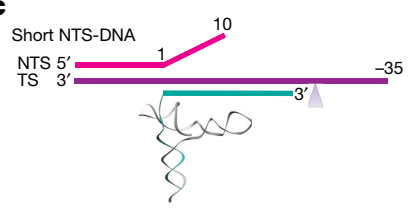

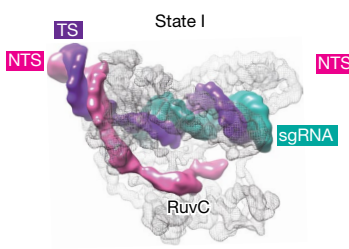
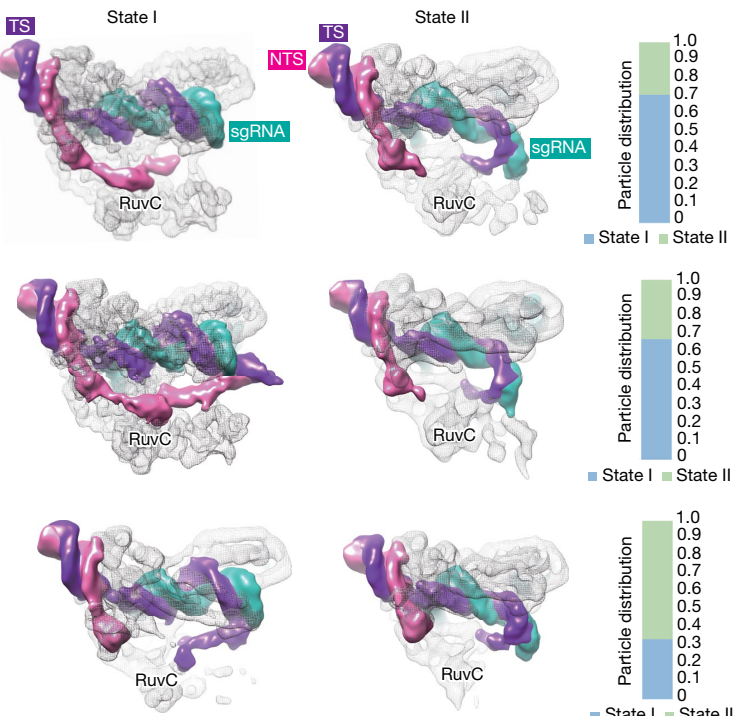

\section{Corrected Fig. 4}

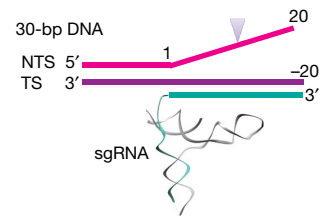

b

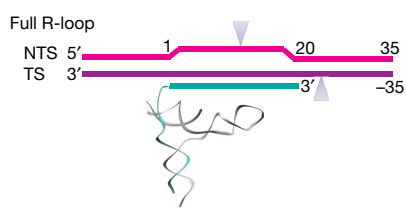

c

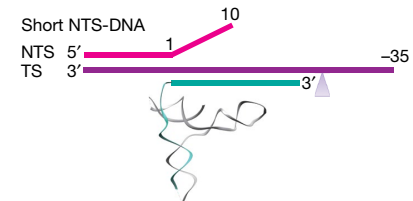

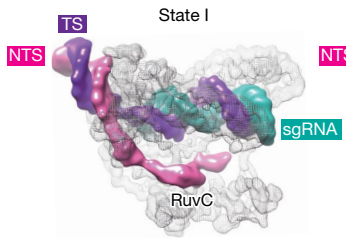
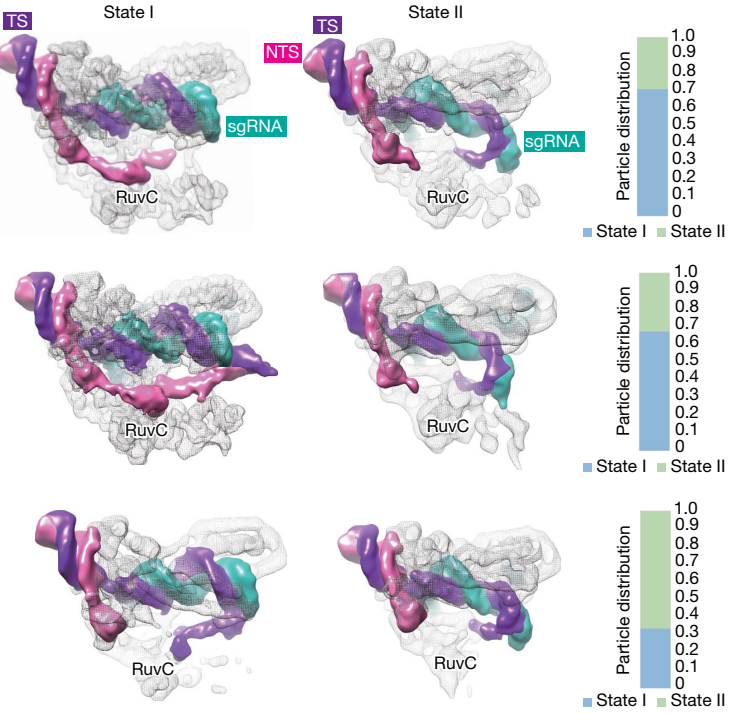

Fig. 2 This is the corrected Fig. 4 and the incorrect Fig. 4 published in the original Article. This is the corrected Fig. 4 and the incorrect Fig. 4 published in the original Article. 
Original Extended Data Fig. 6a

a

DpbCasX

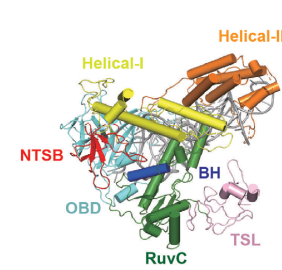

$\downarrow-9$
AacCas12b

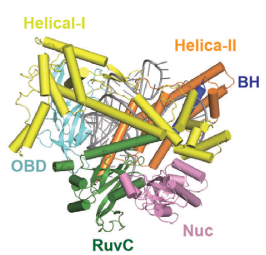

$\downarrow-10$

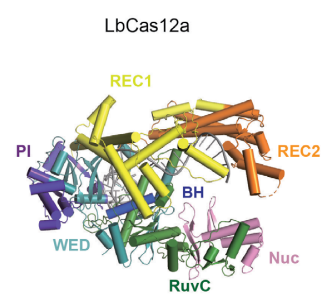

$\downarrow-0$
Original Extended Data Fig. 6e

e

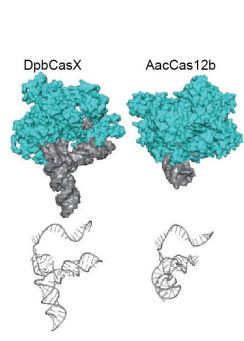

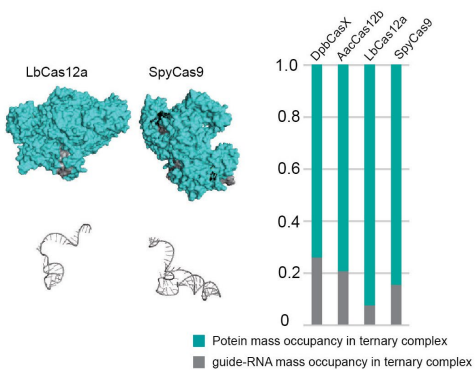

a

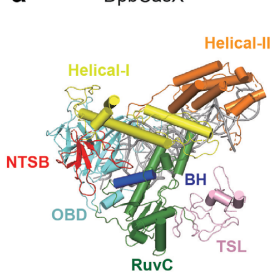

$\downarrow-\hat{U}$
Corrected Extended Data Fig. 6a
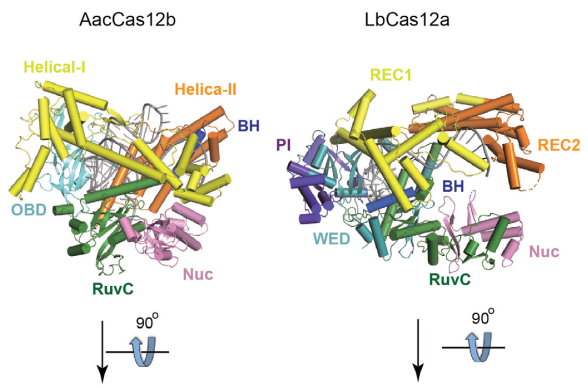

\section{Corrected Extended Data Fig. 6e}

e

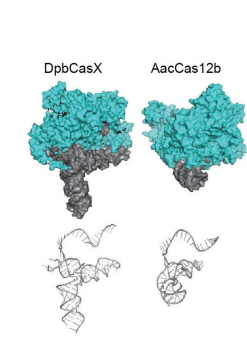

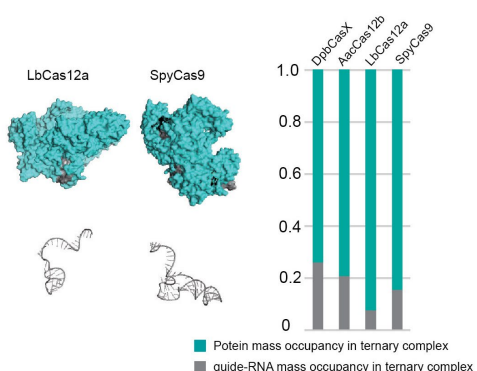

Fig. 3 This is the corrected Extended Data Fig. 6a, e and the incorrect Extended Data Fig. 6a, e published in the original Article. The CasX sgRNA cartoons have been updated. 